\title{
Silicon Sensor Developments for the CMS Tracker Upgrade
}

\author{
Joachim Erfle* on behalf of the CMS tracker collaboration \\ University of Hamburg, Germany \\ E-mail: Joachim.Erfle@desy.de
}

\begin{abstract}
CMS started a campaign to identify the future silicon sensor technology baseline for a new tracker for the high-luminosity phase of the LHC. We ordered a large variety of $6^{\prime \prime}$ wafers in different thicknesses and technologies at HPK. Thicknesses ranging from $50 \mu \mathrm{m}$ to $300 \mu \mathrm{m}$ are explored on floatzone (FZ), magnetic Czochralski (MCz) and epitaxial (Epi) material both in n-in-p and p-in-n versions. Each wafer contains different structures to answer different questions, e.g. geometry, Lorentz angle, radiation tolerance, annealing behaviour, read-out schemes. An overview about these structures will be given, together with first results for a new thinning process and new readout schemes.
\end{abstract}

10th International Conference on Large Scale Applications and Radiation Hardness of Semiconductor Detectors

6-8 July 2011

Florence, Italy

\footnotetext{
* Speaker.
} 


\section{Introduction}

\subsection{Motivation}

It is planned to upgrade the LHC to a High Luminosity-LHC (with an instantaneous luminosity of about $\left.10^{35} \mathrm{~cm}^{-2} \mathrm{~s}^{-1}\right)$. This will cause a higher track occupancy and a much harsher radiation environment (shown in fig. 1). In order to disentangle the high multitude of tracks, sensors with

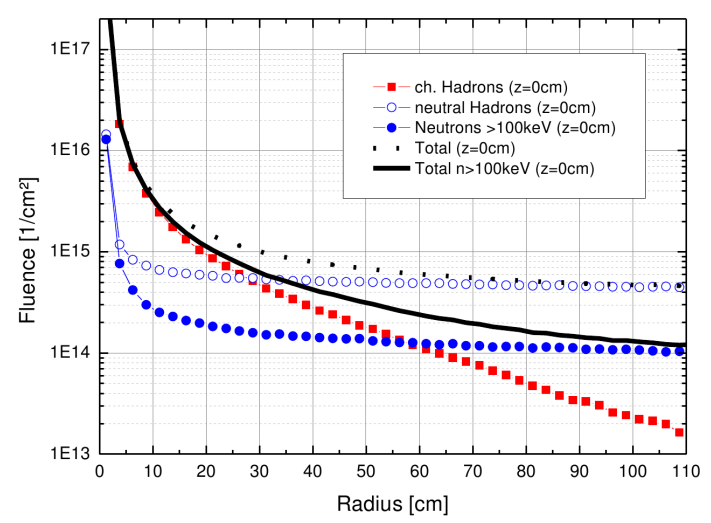

Figure 1: The black line shows the total fluences for different radii in the central region for an integrated luminosity of $3000 \mathrm{fb}^{-1}[1]$.

shorter and more strips are considered. The radiation damages the sensors and increases their leakage current, the depletion voltage and charge carrier trapping. The higher leakage current causes higher noise and more heat load. While the leakage current can be reduced by operating the sensors at lower temperatures, a bigger problem is the increased depletion voltage, making it impossible to fully deplete the sensors at some point. At very large fluences, radiation induced defects cause charge carrier trapping, hereby reducing the signal. Hence it is required to find more radiation hard material and layouts and to increase the granularity of tracking detectors.

In order to define the baseline for the LHC-upgrade a large campaign has been started within CMS with the participation of a significant number of institutes. The aim is to compare different materials and structures from one high quality producer. The main goals of the campaign are to identify the best material, and to find the best sensor layout.

\section{Measurement campaign overview}

\subsection{The materials}

Table 1 gives an overview of the available materials. We have six wafers of each of the three doping types (n-type, $\mathrm{p}$-type with $\mathrm{p}$-spray isolation and p-type with $\mathrm{p}$-stop isolation). That makes 18 wafer of each material and 140 in total. Each wafer contains a number of teststructures (shown in fig. 2), some of which will be introduced below. In order to investigate the radiation-hardness, these structures will be irradiated with neutrons, protons, and both, to fluences corresponding to the expected fluences at specific positions in the future CMS-Tracker (shown in table 2). 


\begin{tabular}{c|c|c|c} 
material & thinning method & active thickness & physical thickness \\
\hline FZ & deep diffusion & $120,200,300 \mu \mathrm{m}$ & $320 \mu \mathrm{m}$ \\
FZ & - & $200 \mu \mathrm{m}$ & $200 \mu \mathrm{m}$ \\
FZ & handling wafer & $120 \mu \mathrm{m}$ & $320 \mu \mathrm{m}$ \\
$\mathrm{MCz}$ & - & $200 \mu \mathrm{m}$ & $200 \mu \mathrm{m}$ \\
Epi & handling wafer & $50,100 \mu \mathrm{m}$ & $320 \mu \mathrm{m}$
\end{tabular}

Table 1: Overview of the materials. With floatzone silicon (FZ), magnetic Czochralski silicon (MCz) and epitaxically grown silicon (Epi).

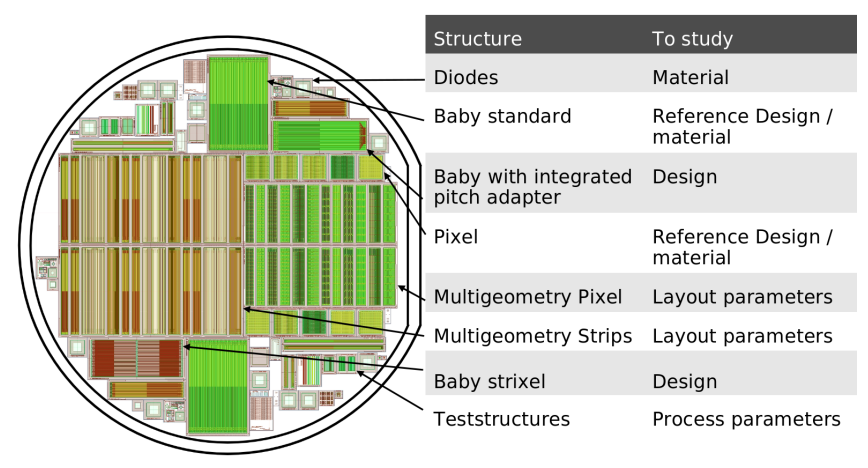

Figure 2: Wafer of the campaign with the different structures.

\begin{tabular}{c|c|c|c|c} 
Radius & Protons $/ 10^{14} \mathrm{neq}_{\mathrm{cm}} \mathrm{c}^{2}$ & Neutrons $/ 10^{14} \mathrm{n}_{\mathrm{eq}} \mathrm{cm}^{2}$ & Total $/ 10^{14} \mathrm{n}_{\mathrm{eq}} \mathrm{cm}^{2}$ & Ratio $\mathrm{p} / \mathrm{n}$ \\
\hline $40 \mathrm{~cm}$ & 3 & 4 & 7 & 0.75 \\
$20 \mathrm{~cm}$ & 10 & 5 & 15 & 2.0 \\
$15 \mathrm{~cm}$ & 15 & 6 & 21 & 2.5 \\
$10 \mathrm{~cm}$ & 30 & 7 & 37 & 4.3 \\
$5 \mathrm{~cm}$ & 130 & 10 & 140 & 13
\end{tabular}

Table 2: This table shows the planned fluence points for the irradiations at the given radii of the future CMS tracker [2].

\subsection{Test structures}

Basic structures are used to understand material and processing parameters. The diodes (shown in fig. 3) are used to understand material characteristics and performance without being influenced by a more complex surface structure. It consists of a single pn-junction with a guard structure on the surface, a back-side contact and the silicon bulk in between. The teststructure (shown in fig. 4) contains different substructures to measure process characteristics on the surface. These structures are used to measure the resistivities and capacitances of the materials used in the production process and how they are influenced by radiation.

The next type of structures are sensors which are used to tune parameters of strip and pixel sensors. These are the so-called multi geometry structures. The multi geometry pixel (shown in 

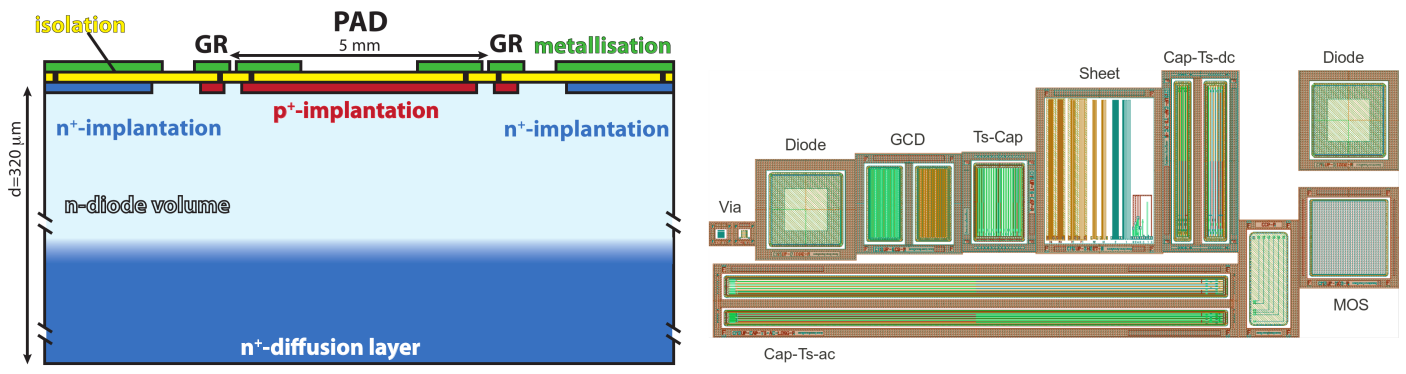

Figure 3: Cut through a diode on deep diffused Figure 4: Teststructure with the different subfloatzone-silicon [3].

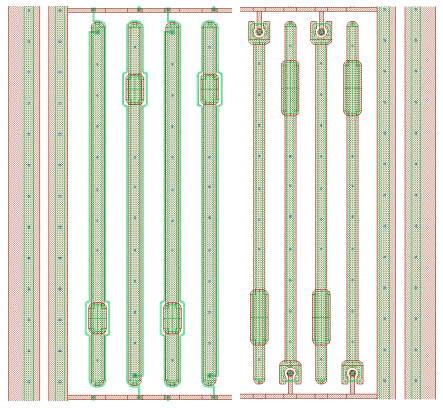

Figure 5: Multi geometry pixel sensor for optimizing layout parameters. structures for process qualification.
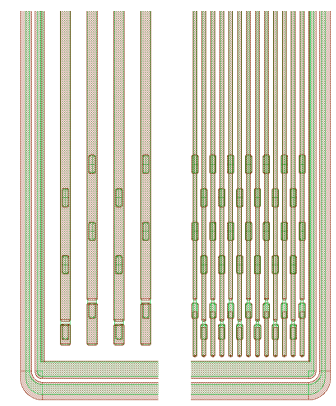

Figure 6: Multi geometry strip sensor for optimizing layout parameters.

fig. 5) contains 12 different pixel-variations, split up in 2 different bias-technologies, 3 different width/pitch-ratios and 2 different pixel length. The multi geometry strips (shown in fig. 6) also splits up in 12 different strip regions, with 4 different pitches and 12 different widths and metal overhangs.

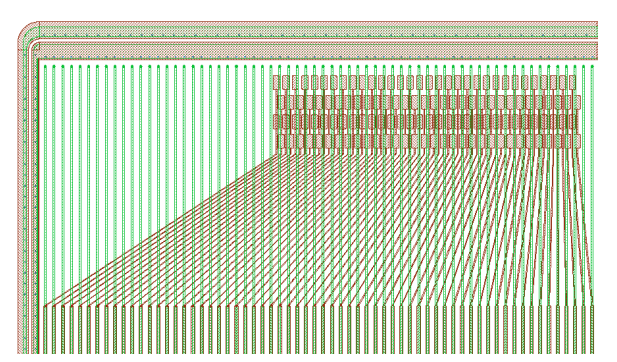

Figure 7: Baby sensor with metal routing integrated in first metal layer.

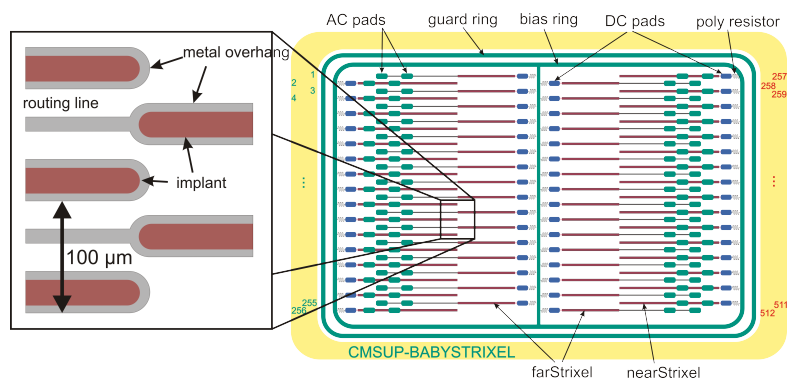

Figure 8: Short strip sensor with routing of the inner strips in the first metal layer.

Structures for testing new layouts are used to test for example possibilities to do routing on the first metal layer. This could be used to connect readout to strips, when having more than 2 strips in a row. One structure consists of a baby sensor with a fan-in with bond pads on one side (shown in fig. 7). To be able to collect charge in the fan-in area the implant extends into it. The other structure is a strixel sensor with 4 short strips (shown in fig. 8) in a row. In this design, the readout would be placed on the sensor edges, the near strixels (in the outer sensor region) will be 
contacted directly through the AC Pads, while the signal from the far strixels (in the inner sensor region) is routed to the edge by a thin metal line which also provides contact through the bond pads. Alternatively, some samples are produced with a dedicated second metal layer for the routing.

\subsection{Measurements}

The most important measurements, which are performed on nearly all of the structures, are IV/CV (Current vs Voltage/Capacitance vs Voltage) measurements for understanding the material basics, TCT (Transient Current Technique) measurements for investigating charge collection in silicon in a time resolved manner, resistivity and capacitance measurements at operating voltage for understanding the structure parameters and CCE (Charge Collection Efficiency) measurements using the alibava-readout system [5] for investigating the response of a sensor to the signal induced by a laser or a radioactive source.

\section{First results}

\subsection{Deep diffused material}

Deep diffusion is a new method to produce detectors with a smaller active than physical thickness. This is done by diffusion of a high number of dopants deep into the material. The standard

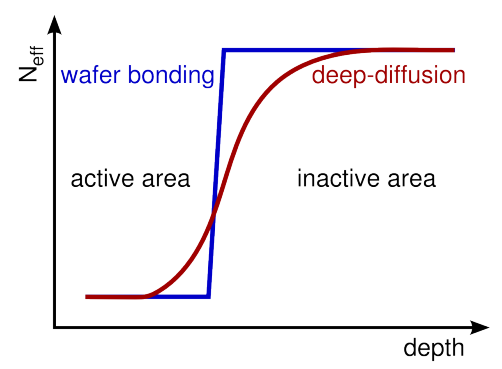

Figure 9: Interface shape of deep diffusion vs wafer bonding.

method is to bond an active wafer to a handling wafer, which is more expensive but creates a harder interface between active and inactive volume than using deep diffusion (as illustrated in figure 9). To inactivate part of the wafer by deep diffusion, a high number of dopants is diffused in from the backside over a depth of up to $200 \mu \mathrm{m}$. Since diodes behave like parallel plate capacitors in reverse bias, the depletion depth and the charge carrier concentration can be calculated from the capacitance versus voltage measurement (shown in fig. 10) using $N_{\text {eff }}=\frac{(2 \Delta V) /\left(\Delta\left(1 / C^{2}\right)\right)}{q \cdot \mathcal{E} \cdot A^{2}}$ and depth $d=\frac{\varepsilon \cdot A}{C}$. In fig. 11 such a concentration versus depth profile is shown. It is clearly visible that the thinner diodes have a much softer interface. They show a much higher number of dopants in the interface area and have also some defects introduced by deep diffusion [3]. Nevertheless the number of defects is not as high as the expected number from radiation.

\subsection{Small irradiation step for deep diffused material}

In this first irradiation step the deep diffusion technology was tested. Therefore one large and one small diode of $\mathrm{p}$ - and n-type silicon and the three thicknesses were irradiated to a fluence of 


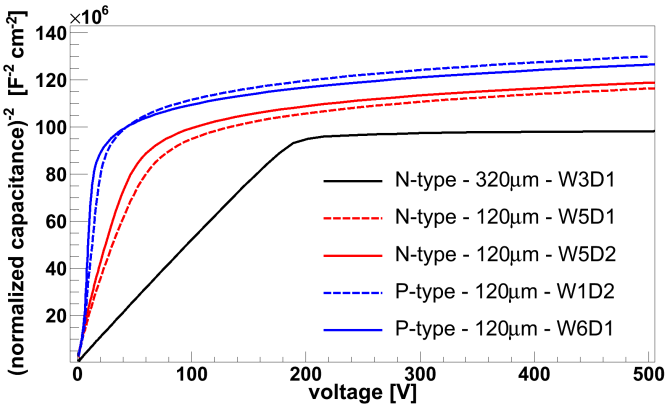

Figure 10: Capacitance voltage characteristics of different deep diffused materials.
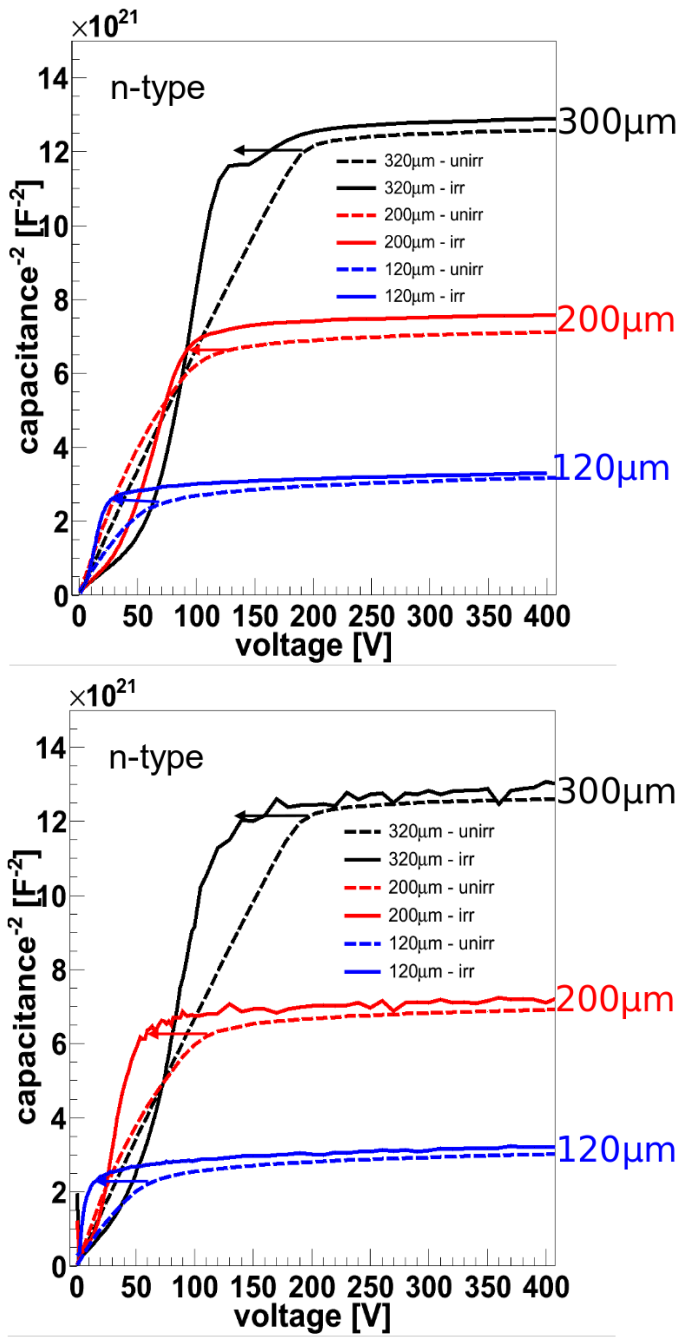

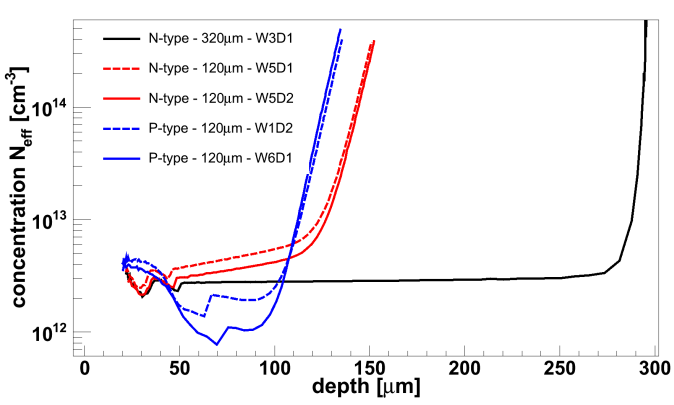

Figure 11: Profile of effective charge carrier concentration, calculated from fig. 10 .
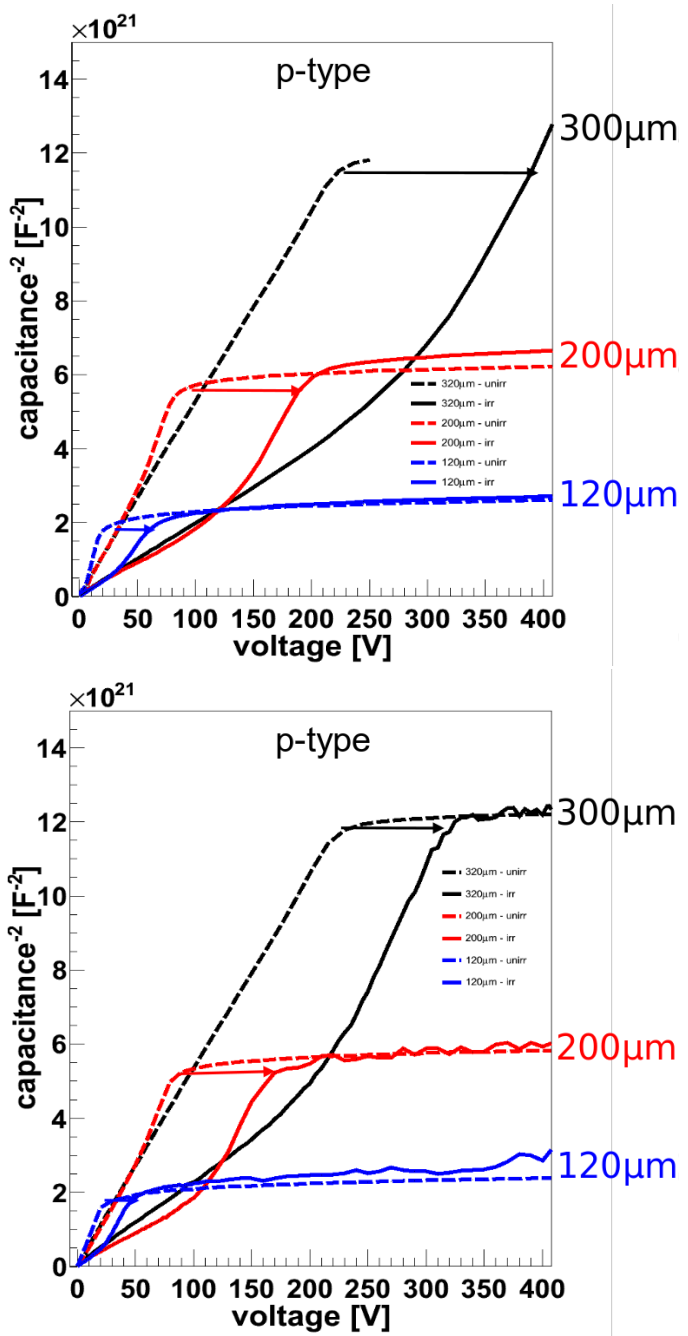

Figure 12: Capacitance voltage characteristics for different thicknesses and materials, before and after irradiation with neutrons (upper plots) and protons (lower plots).

$10^{14} \mathrm{neq}_{\mathrm{em}} \mathrm{cm}^{-2}$ with reactor neutrons and protons (25 MeV), respectively. Figures 12 show that the p-type material has a higher depletion voltage, whereas the n-type did undergo type-inversion and 
has a lower depletion voltage. However, the features that were introduced by the deep diffusion are not dominating anymore.

\subsection{Sensor with integrated pitch adapter}
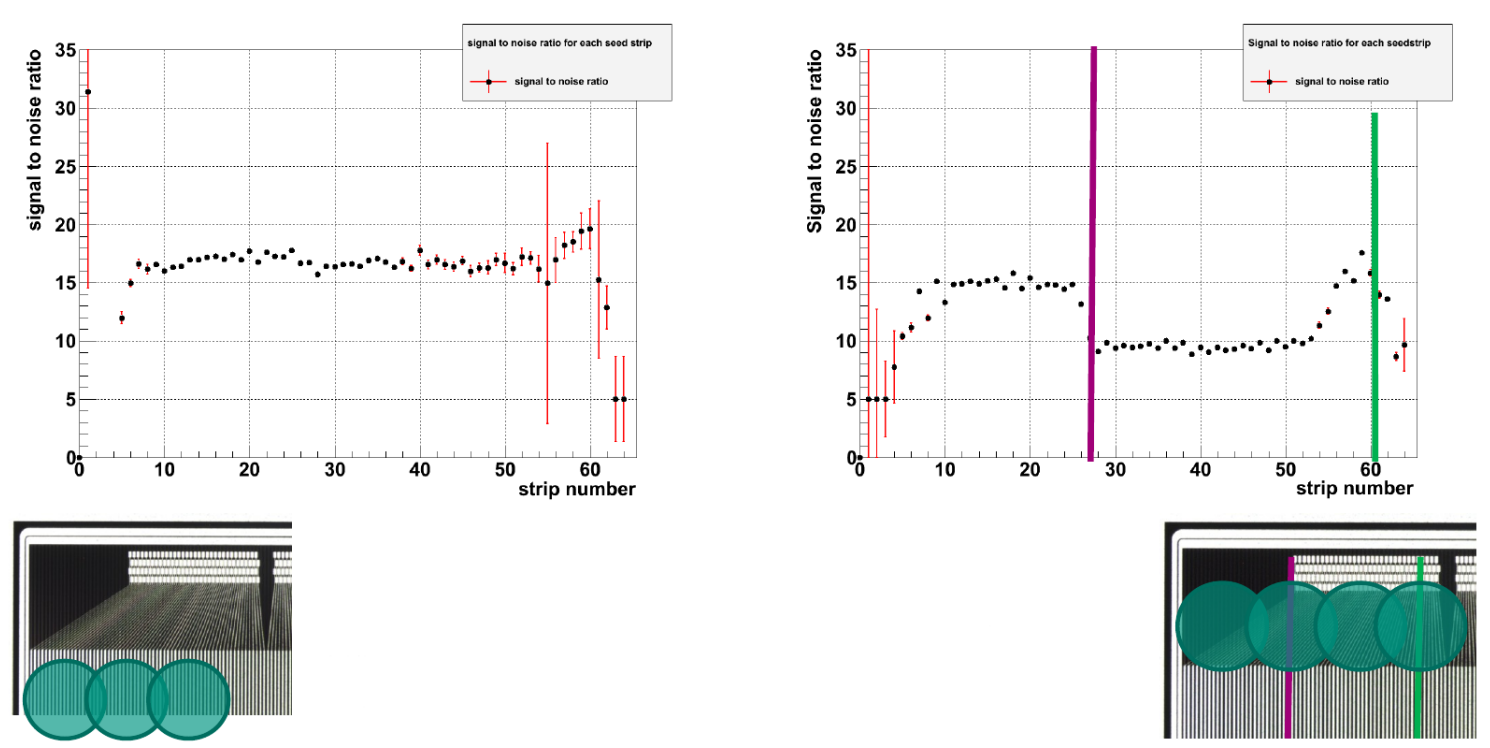

Figure 13: Signal-to-noise ratio of a structure with integrated pitch adapter. On the left plot the source is above the normal region, on the right plot the source is above the pitch adapter region[4].

Signal-to-noise measurements were performed in order to test sensors with integrated pitch adapters (shown in fig. 13) using the alibava system and a collimated ${ }^{90} \mathrm{Sr}$-source which was moved across the sensor to get signal in different regions. It is observed that the signal in the standard strip region and in the outer region of the pitch adapter is good, whereas below the metal routing of the pitch adapter the signal is reduced, while the noise is constant. This results in a reduced signal-to-noise ratio.

\subsection{Short strip sensor with first metal routing}

In fig. 14 the signal induced by a laser is shown versus laser-position for different readout strips. On the left plot, signal is created in the inner region (the far strips). In that region the signal is as expected. It is on the left or right strip, depending on the laser position. On the right plot, however, the far strip also shows some signal from the near area. The hypothesis is, that the metal routing also collects charge. Future measurements will be needed to verify that. 


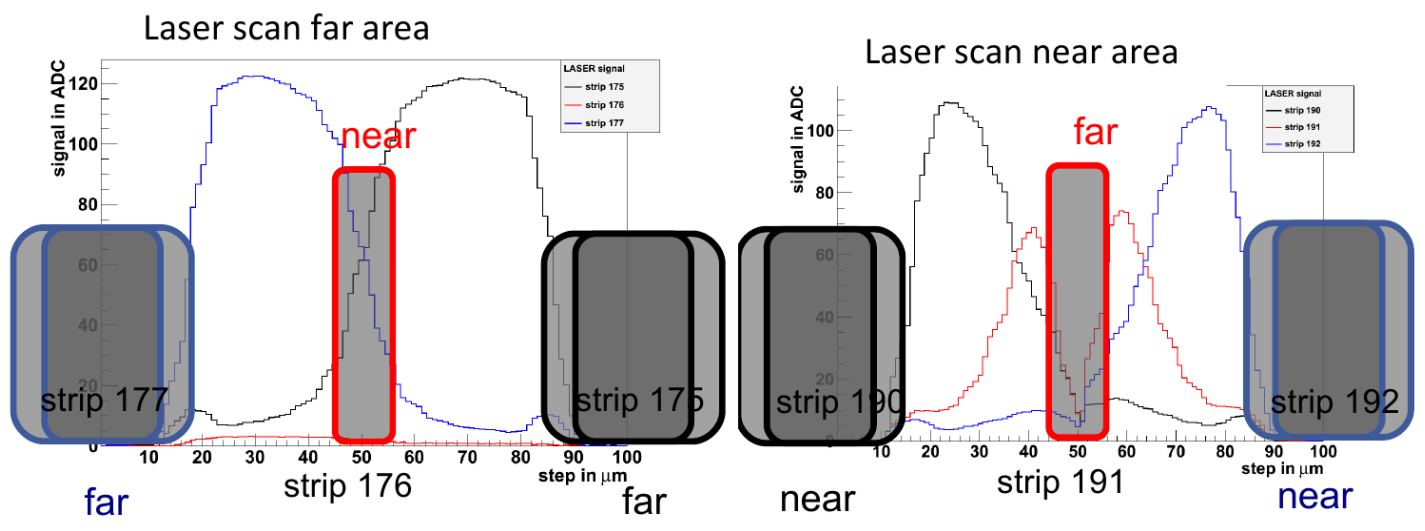

Figure 14: Signal of a short strip structure. On the right the laser scan was performed in the near area, on the left it was performed in the far area. The boxes symbolise the strips whose signal is shown. The outer strips are the ones, that should show the signal, the middle one should not [4].

\section{Conclusions}

In the future, we will need materials and designs that can cope with the radiation levels and the occupancy at the upgraded LHC. A campaign was therefore started to measure a big set of different structures and materials of one high quality producer to define the baseline for the sensors of the HL-LHC upgrade. All the materials are working and the standard structures give good results. The new layouts are also working, but it has to be investigated further, if such layouts could be used. Data of materials being irradiated to higher fluences will reveal which material and design is most suitable for the tracker for the high luminosity LHC.

\section{References}

[1] S. Müller, The Beam Condition Monitor 2 and the Radiation Environment of the CMS Detector at the LHC, Karlsruhe Institute of Technology, 2011, IEKP-KA/2011-1, CMS TS-2010/042

[2] A. Dierlamm, Silicon Sensor Developments for the CMS Tracker Upgrade, PSD9, Aberystwyth, 2011

[3] A. Junkes, Influence of radiation induced defect clusters on silicon, particle detectors, Ph.D. thesis, Hamburg University, DESY-THESIS-2011-031 (July 2011)

[4] A. Kornmayer, Untersuchungen zur Signalkopplung an neuartigen Siliziumstreifensensorgeometrien, Karlsruhe Institute of Technology, 2011, IEKP-KA/2011-17

[5] R. Marco-Hernández and ALIBAVA COLLABORATION, A Portable Readout System for Microstrip Silicon Sensors (ALIBAVA), IEEE TRANSACTIONS ON NUCLEAR SCIENCE, VOL. 56, NO. 3 , JUNE 2009 